\title{
Evaluation of $\mathrm{AJCC}$ and an Alternative Tumor Classification System for Primary Vulvar Squamous Cell Carcinoma
}

\author{
Sarah T. Le, MBBS (Hons), BMedSci (Hons)a, ${ }^{\mathrm{a}}$; Pritesh S. Karia, MPH; \\ Beverley J. Vollenhoven, MBBS (Hons), $\mathrm{PhD}^{\mathrm{c}}$; Robert J. Besaw, $\mathrm{MPH}^{\mathrm{a}}$; Colleen M. Feltmate, MD; \\ and Chrysalyne D. Schmults, MD, MSCE ${ }^{a}$
}

\begin{abstract}
Background: Currently, no studies have attempted to validate the AJCC tumor (T) class for vulvar cancer or examine its performance via clinical data. The goal of this study was to identify risk factors associated with poor outcomes in vulvar squamous cell carcinoma (vSCC) and compare prognostic discrimination of these outcomes between the AJCC T-classification system and the newly developed Brigham and Women's Vulvar Tumor Classification system (BWVTC). Methods: A 15-year, 2-center retrospective cohort study of primary vSCCs (N=226) was undertaken. Risk factors for poor outcomes, including local recurrence (LR), nodal and distant metastasis (NM and DM, respectively), disease-specific death (DSD), and overall death (OD) were determined using competing risks models. Poor outcomes were analyzed by $T$ stage with regard to each classification system's distinctiveness, homogeneity, and monotonicity. Results: AJCC T stages were indistinct, with overlapping $95 \%$ confidence intervals for 10-year cumulative incidences of poor outcomes. Most poor outcomes occurred in low AJCC T stages: $\mathrm{T} 1 \mathrm{a} / \mathrm{T} 1 \mathrm{~b}$ contained $77 \%$ of $\mathrm{LR}, 79 \%$ of NM, $66 \%$ of DM/DSD, and $78 \%$ of OD, indicating poor homogeneity and monotonicity. Five risk factors were independent predictors of poor outcomes: history of lichen sclerosus, tumor diameter $\geq 2.0 \mathrm{~cm}$, tumor depth $\geq 3.0 \mathrm{~mm}$, poor differentiation, and mucosal involvement, and these were used to develop the BWVTC (BWVTC BWT1 = 0 risk factors; BWT2 = 1 risk factor; BWT3 = 2 risk factors; and BWT4 = $\geq 3$ risk factors). The BWVTC displayed superior homogeneity and monotonicity, with most poor outcomes occurring in high T stages: T3/T4 contained $87 \%$ of LR, $92 \%$ of NM, $91 \%$ of DM/DSD, and $78 \%$ of OD $(P<.001)$, although not all $T$ stages were statistically distinct in this small cohort. Conclusions: The BWVTC offers improved prognostic discrimination over the AJCC T-classification system. Validation in population-based cohorts and in vulvar cancers other than SCC is needed.
\end{abstract}

J Natl Compr Canc Netw 2018;16(1):42-49 doi: 10.6004/jnccn.2017.7022

\section{Background}

Vulvar cancer is the fourth most common gynecologic malignancy, accounting for $5 \%$ of female genitalia tumors. ${ }^{1}$ Vulvar squamous cell carcinoma (vSCC) constitutes $>90 \%$ of vulvar cancer. ${ }^{2}$ In the United States, the age-adjusted incidence of vSCC is 2.5 per 100,000 women. ${ }^{3}$ The most significant prognostic factor for vSCC is lymph node status. ${ }^{4,5}$ Other factors associated

From the aDepartment of Dermatology, Brigham and Women's Hospital, Harvard Medical School, Boston, Massachusetts; 'bedicine, Nursing and Health Sciences, Monash University, Melbourne, Australia; 'Department of Obstetrics and Gynecology, Monash University, Melbourne, Australia; and 'Department of Obstetrics and Gynecology, Brigham and Women's Hospital, Harvard Medical School, Boston, Massachusetts.

Submitted March 13, 2017; accepted for publication August 3, 2017.

The authors have disclosed that they have no financial interests, arrangements, affiliations, or commercial interests with the manufacturers of any products discussed in this article or their competitors. with poor outcomes include age, tumor diameter, tumor depth, tumor class, histologic grade, lymphovascular space invasion, margin distance, and clitoral involvement. $^{6-14}$ Due to inconsistent definitions and results across studies, most risk factors have not been adopted into current staging systems.

The vulvar cancer staging system in the 8 th edition of the AJCC Staging Manual is used for vSCC, but also

Author contributions: Dr. Le had full access to all the data in the study and takes responsibility for the integrity of the data and the accuracy of the analysis. Study concept and design: Le, Vollenhoven, Schmults. Data acquisition: Le, Karia, Besaw. Data analysis and interpretation: Le, Karia, Schmults. Manuscript preparation: Le. Critical revision: All authors. Statistical analysis: Le. Administrative, technical, or material support: Le, Karia, Besaw. Study supervision: Schmults.

Correspondence: Chrysalyne D. Schmults, MD, MSCE, Department of Dermatology, Brigham and Women's Hospital, 1153 Centre Street, Suite 4J, Boston, MA 02130. E-mail: cschmults@bwh.harvard.edu 
Tumor Classification System for Vulvar SCC

for rare cancer types occurring on the vulva, such as verrucous carcinoma, vulvar Paget disease, adenocarcinoma, basal cell carcinoma, and Bartholin gland carcinoma (Table 1). ${ }^{15}$ AJCC uses the TNM staging system based on tumor $(\mathrm{T})$, nodal $(\mathrm{N})$, and metastatic (M) parameters. Nodal criteria have been well refined and validated over the past few decades, but there has been minimal focus on $\mathrm{T}$ classification, even though most patients present with localized disease confined to the vulva. ${ }^{3}$ No studies have attempted to validate the AJCC T classification for vulvar cancer or examine its performance via clinical data.

This study was undertaken to evaluate the current AJCC vulvar cancer T-classification system with regard to vSCC. Study data were used to identify risk factors independently associated with poor outcomes in vSCC. An alternative T-classification system, the Brigham and Women's Vulvar Tumor Classification (BWVTC) system, was developed based on these prognostic factors (Table 2) and compared against the AJCC T-classification system.

\section{Methods}

\section{Patient Selection}

After approval from the Partners Human Research Committee, the Research Patient Data Registry (RPDR), a centralized clinical registry, was searched for malignant neoplasms of the vulva diagnosed from January 1, 1995, to December 31, 2009, at Brigham

\begin{tabular}{|c|c|}
\hline $\begin{array}{l}\text { Tumor } \\
\text { Classification }\end{array}$ & Definition \\
\hline T1a & $\begin{array}{l}\text { Tumor } \leq 2 \mathrm{~cm} \text { and stromal invasion } \leq 1.0 \mathrm{~mm} \text {, } \\
\text { confined to the vulva or perineum }\end{array}$ \\
\hline T1b & $\begin{array}{l}\text { Tumor }>2 \mathrm{~cm} \text { or stromal invasion }>1.0 \mathrm{~mm} \text {, } \\
\text { confined to the vulva or perineum }\end{array}$ \\
\hline $\mathrm{T} 2$ & $\begin{array}{l}\text { Tumor of any size with extension to adjacent } \\
\text { perineal structures (lower/distal one-third } \\
\text { urethra, lower/distal one-third vagina, anal } \\
\text { involvement) }\end{array}$ \\
\hline T3 & $\begin{array}{l}\text { Tumor of any size with extension to any of } \\
\text { the following - upper/proximal two-thirds of } \\
\text { urethra, upper/proximal two-thirds vagina, } \\
\text { bladder mucosa, or rectal mucosa-or fixed to } \\
\text { pelvic bone }\end{array}$ \\
\hline
\end{tabular}

Modified with permission of American Joint Committee on Cancer (AJCC), from Amin MB, Edge S, Greene F, et al. AJCC Cancer Staging Manual, 8th ed. New York, NY: Springer; 2017:636; permission conveyed through Copyright Clearance Center, Inc.

\begin{tabular}{|c|c|}
\hline Tumor Classification & Definition \\
\hline BWT1 & 0 high-risk factors ${ }^{a}$ \\
\hline BWT2 & 1 high-risk factor \\
\hline BWT3 & 2 high-risk factors \\
\hline BWT4 & $\geq 3$ high-risk factors \\
\hline
\end{tabular}

Abbreviation: BWT, Brigham and Women's tumor class.

aHigh-risk factors include history of lichen sclerosus, tumor diameter $\geq 2.0 \mathrm{~cm}$, tumor depth $\geq 3.0 \mathrm{~mm}$, poorly differentiated histology, and mucosal epithelial involvement.

and Women's Hospital and Massachusetts General Hospital. Diagnoses were based on ICD-9 codes.

Tumor location was stratified into categories of cutaneous, mucosal, or both. Classification was based on pathology reports, but if not specified, the anatomic location recorded in clinical notes was used. Tumors involving the labia majora, mons pubis, clitoral hood, and perineal body were classified as cutaneous. Tumors with involvement of the labia minora, clitoris, urethra, vaginal vestibule, Bartholin gland, introitus (entrance to the vaginal canal), and posterior fourchette were classified as mucosal. Tumors were classed according to the 8th edition AJCC vulvar cancer Tclassification guidelines. Outcomes of interest were also recorded, including local recurrence (LR), nodal metastasis (NM), distant metastasis (DM), diseasespecific death (DSD), and overall death (OD).

\section{Statistical Analysis}

Baseline patient and tumor characteristics were summarized using frequency tabulation. Cox proportional hazards models with the Fine-Gray method for the competing risk of non-vSCC death were used for univariable and multivariable analyses of risk factors associated with LR, NM, DM, DSD, and OD. ${ }^{16}$ Separate multivariable models were built for each outcome of interest by incorporating risk factors that were borderline or statistically significant $(P \leq .20)$ on univariable analysis. This was followed by backward stepwise elimination of variables with small prognostic effects $(P>.10)$. Multivariable prognostic factors predictive of at least one poor outcome of interest (LR, NM, DM, DSD, and OD) were used to develop the BWVTC system. Once independent prognostic factors were determined from multivariable modeling, different combinations and accumulations of risk factors were evaluated in terms of distinctiveness/ 
homogeneity/monotonicity to determine the system with the best risk stratification (prognostic groupings without overlapping confidence intervals [CIs]). All risk factors were weighted evenly, because in this small initial study, hazard ratio (HR) CIs were not sufficiently narrow to take differences in hazards into account for different risk factors.

Survival time for each poor outcome was recorded as the time between primary vSCC tumor diagnosis and outcome arising from the tumor. The censoring date for patients who did not develop an outcome of interest was defined as the date of last follow-up from the medical records (except for OD, for which the date of death as per last clinic note or the Social Security Death Index was used).

The AJCC and BWVTC T-classification systems were evaluated for system distinctiveness, homogeneity, and monotonicity. Distinctiveness (outcome differences between classes) was assessed by comparing the 10-year cumulative incidences and 95\% CIs for each outcome of interest between $\mathrm{T}$ classes. Homogeneity (outcome similarity within classes) was evaluated by comparing the proportion of poor outcomes occurring in low T classes between AJCC and BWVTC. Monotonicity (outcome worsening with increasing class) was assessed by comparing the proportion of poor outcomes occurring in high $\mathrm{T}$ classes between AJCC and BWVTC. McNemar test was used to compare the homogeneity and monotonicity between the $2 \mathrm{~T}$-classification systems. Life tables and Kaplan-Meier curves were constructed to demonstrate the event-free survival for disease-related and overall survivals according to each T class. Pair-wise comparison (log-rank test) was conducted on adjacent curves within each figure to determine whether there was a significant difference between the $T$ classes. All statistical analyses were performed using STATA 12.0 (StataCorp LP, College Station, TX), using a 2 -sided $5 \%$ type I error rate.

\section{Results}

The RPDR search yielded 298 cases of invasive (non-in situ) vSCC. After medical records review, 72 patients had NM at diagnosis and were excluded, leaving 226 patients with primary vSCCs in the final study cohort.

Baseline patient and tumor characteristics are summarized in supplemental eTable 1 (available with this article at JNCCN.org). The mean age at diagnosis was 66 years (SD, 15 years), and the median follow-up time was 59 months (interquartile range, 16-79 months). Most patients (87\%) were nonHispanic white and very few were immunosuppressed (6\%). Nearly half (47\%) of the patients had a smoking history and two-thirds (63\%) had a history of vulvar intraepithelial neoplasia. A small proportion of patients had a history of genital warts (10\%), human papillomavirus infection $(8 \%)$, or transmitted infection (5\%). More than one-third of the patients had a history of lichen sclerosus (36\%); 30\% were diagnosed with a second primary malignancy either prior to vSCC diagnosis or during the follow-up period. Patients with a second primary malignancy had a lower risk of developing a poor outcome from vSCC compared with those without a secondary tumor (HR, 0.70; 95\% CI, 0.43-1.15). The competing risk analysis adjusts for this competing risk of non-vSCC death prior to development of study end points.

Mean tumor diameter was $2.3 \mathrm{~cm}(\mathrm{SD}, 1.9 \mathrm{~cm})$, and average tumor depth was $5.2 \mathrm{~mm}(\mathrm{SD}, 6.3 \mathrm{~mm})$. Most tumors were moderately differentiated (90\%), with mucosal involvement (69\%), and without perineural invasion (96\%) or lymphovascular invasion (94\%). Most tumors were primarily resected (81\%), and most adjuvant therapy was used in higher T-class cases (see supplemental eTable 2). Of patients undergoing excision, $15 \%$ had a positive or close margin, $76 \%$ had clear margins, and $2 \%$ were unspecified. Patients with positive or close margins were treated with further resection $(70 \%)$ or salvage radiation therapy $(30 \%)$.

Nodal evaluation had been performed in all patients to confirm NO status. Evaluation was via clinical or radiologic assessment of lymph nodes $(n=78)$, sentinel node biopsy $(n=3)$, or lymphadenectomy (LAN; $n=145)$. Patients with high-stage tumors (BWT3/BWT4) were significantly more likely to undergo LAN (69\% [107/156] of patients with highstage BWVTC tumors underwent LAN vs $54 \%$ [38/70] of those with low-stage tumors; $P=.04]$. LAN was associated with better outcomes in patients with high-stage tumors. Those with BWVTC BWT3/ BWT4 tumors who underwent LAN had a lower risk of NM (BWT3, 11\% and BWT4, 8\%) compared with those not having LAN (BWT3, 15\% and BWT4, $31 \% ; P=.036)$, and also had lower risks of DM/DSD (with LAN: BWT3, 7\% and BWT4, 19\% vs with- 
Tumor Classification System for Vulvar SCC

out LAN: BWT3, 30\% and BWT4, 38\%; P=.001). In patients with low-stage BWVTC tumors (BWT1/ BWT2; $n=70$ ), only 3 cases progressed to metastasis and death, and therefore LAN had no demonstrable impact on outcomes (although approximately half of these patients underwent LAN).

Table 3 shows the 10-year cumulative incidence of outcomes of interest by AJCC class and the proportion of outcomes occurring in low- and high-stage classes. There were 65 cases (29\%) of LR, 24 (11\%) of NM, 35 (15\%) of DM or DSD, and 99 (44\%) of OD. Among the $33 \%$ of patients who developed disease recurrence (LR/NM/DM), the primary tumor was treated with surgery alone (79\%), adjuvant therapy alone (7\%), or surgery plus adjuvant therapy $(14 \%)$. A sensitivity analysis showed no significant difference in disease recurrence by treatment modality of the primary tumor. Most poor outcomes occurred within 5 years of diagnosis (LR, 85\%; NM, 88\%; DM/DSD, 81\%; OD, $57 \%$ ), although 3 patients experienced a poor outcome $\geq 10$ years after diagnosis (LR, 150 months; DM, 137 months; DSD, 181 months). The patient with LR had multiple medical comorbidities and declining mental status, which delayed the diagnosis of recurrent disease. The patients with DM and DSD both experienced years of LR disease before the occurrence of disseminated disease and death, respectively.

Most tumors were AJCC T1b (68\%), few tumors were AJCC T1a (16\%) and T2 (14\%), and 4 patients (2\%) presented with AJCC T3 tumors. CIs for AJCC $\mathrm{T}$ classes overlapped substantially for all end points, indicating that the AJCC T classes are not distinct. Most poor outcomes occurred in low AJCC T classes.
Specifically, T1a/T1b contained $77 \%$ of the LR, $79 \%$ of NM, $66 \%$ of DM or DSD, and 78\% of OD. The low $\mathrm{T}$ classes thus comprised a heterogeneous mix of cases having mostly good outcomes, but also contained the large majority of those having poor outcomes, indicating poor homogeneity and monotonicity.

Results of the multivariate analysis of risk factors associated with the development of LR, NM, DM or DSD, and OD are summarized in Table 4. History of lichen sclerosus (HR, 1.68; 95\% CI, 1.01-2.77), poor tumor differentiation (HR, 1.85; 95\% CI, 0.94-3.65), tumor diameter $\geq 2.0 \mathrm{~cm}$ (HR, 1.70; 95\% CI, 0.982.96), and any mucosal epithelial involvement (HR, 2.02; 95\% CI, 1.08-3.78) were associated with an increased risk of LR. Tumor depth $\geq 3.0 \mathrm{~mm}$ was associated with an increased risk of LR (HR, 2.12; 95\% CI, 1.20-3.77), NM (HR, 4.16; 95\% CI, 1.57-11.04), DM or DSD (HR, 2.47; 95\% CI, 1.13-5.41), and OD (HR, 2.73; 95\% CI, 1.77-4.21). In a subgroup analysis, the excision margin of the primary tumor was not significantly associated with outcomes: LR (HR, 1.01; 95\% CI, 1.00-1.03), NM (HR, 1.00; 95\% CI, 0.941.06), DM or DSD (HR, 1.00; 95\% CI, 0.95-1.05), and OD (HR, 1.00; 95\% CI, 0.98-1.03). In particular, an analysis of tumor excision margin $<8$ versus $\geq 8 \mathrm{~mm}$ was not predictive of any poor outcomes (in the 117 cases for which margin size was specified). A sensitivity analysis including treatment with or without LAN and treatment modality as a variable in the multivariable model did not substantially change HRs or other significant prognostic factors, and therefore it was not incorporated into the prognostic model.

\section{Table 3. Evaluation of the Distinctiveness of the AJCC and BWVTC Classification Systems}

\begin{tabular}{|c|c|c|c|c|c|c|c|c|c|}
\hline \multirow[b]{2}{*}{ Tumor Classification } & \multirow[b]{2}{*}{$\mathbf{N}$} & \multicolumn{2}{|c|}{ LR $(\mathrm{N}=65)$} & \multicolumn{2}{|c|}{ NM $(\mathrm{N}=24)$} & \multicolumn{2}{|c|}{ DM or DSD $(\mathrm{N}=35)$} & \multicolumn{2}{|c|}{$\mathrm{OD}(\mathrm{N}=99)$} \\
\hline & & 10-y CIN & $95 \% \mathrm{Cl}$ & 10-y CIN & $95 \% \mathrm{Cl}$ & 10-y CIN & $95 \% \mathrm{Cl}$ & 10-y CIN & $95 \% \mathrm{Cl}$ \\
\hline \multicolumn{10}{|l|}{ AJCC } \\
\hline T1a & 36 & $15 \%$ & $4-30$ & $3 \%$ & $0-14$ & $9 \%$ & $1-25$ & $47 \%$ & $23-68$ \\
\hline $\mathrm{T} 1 \mathrm{~b}$ & 154 & $48 \%$ & $36-59$ & $20 \%$ & $11-31$ & $24 \%$ & $15-35$ & $61 \%$ & $50-70$ \\
\hline $\mathrm{T} 2$ & 32 & $59 \%$ & $36-77$ & $30 \%$ & $9-54$ & $44 \%$ & $22-64$ & $67 \%$ & $45-82$ \\
\hline T3 & 4 & $50 \%$ & $0-91$ & $0 \%$ & - & $67 \%$ & $5-95$ & $75 \%$ & $12-96$ \\
\hline \multicolumn{10}{|l|}{ BWVTC } \\
\hline BWT1 & 20 & $10 \%$ & $1-33$ & $0 \%$ & - & $10 \%$ & $1-33$ & $51 \%$ & $13-80$ \\
\hline BWT2 & 50 & $24 \%$ & $10-40$ & $5 \%$ & $1-14$ & $5 \%$ & $1-15$ & $54 \%$ & $34-70$ \\
\hline BWT3 & 65 & $30 \%$ & $17-44$ & $17 \%$ & $8-30$ & $19 \%$ & $9-32$ & $42 \%$ & $28-56$ \\
\hline BWT4 & 91 & $76 \%$ & $58-88$ & $32 \%$ & $15-50$ & $44 \%$ & $27-59$ & $76 \%$ & $63-85$ \\
\hline
\end{tabular}

Abbreviations: BWT, Brigham and Women's tumor class; BWVTC, Brigham and Women's Vulvar Tumor Classification system; CIN, cumulative incidence; DM, distant metastasis; DSD, disease-specific death; LR, local recurrence; NM, nodal metastasis; OD, overall death. 
Le et al

\begin{tabular}{|c|c|c|c|c|c|c|c|c|}
\hline \multirow[b]{2}{*}{ Variable } & \multicolumn{2}{|l|}{ LR } & \multicolumn{2}{|l|}{ NM } & \multicolumn{2}{|c|}{ DM or DSD } & \multicolumn{2}{|l|}{ OD } \\
\hline & HR (95\% Cl) & $P$ Value & HR $(95 \% \mathrm{Cl})$ & $P$ Value & HR $(95 \% \mathrm{Cl})$ & $P$ Value & HR $(95 \% \mathrm{Cl})$ & $P$ Value \\
\hline $\begin{array}{l}\text { History of lichen } \\
\text { sclerosus }\end{array}$ & $1.68(1.01-2.77)$ & .04 & $1.58(0.69-3.63)$ & .28 & $1.46(0.75-2.84)$ & .27 & $1.05(0.71-1.58)$ & .79 \\
\hline $\begin{array}{l}\text { Poorly } \\
\text { differentiated } \\
\text { tumor histology }\end{array}$ & $1.85(0.94-3.65)$ & .08 & $2.92(1.00-8.51)$ & .05 & $1.85(0.82-4.18)$ & .14 & $1.25(0.73-2.13)$ & .42 \\
\hline Diameter $\geq 2.0 \mathrm{~cm}$ & $1.70(0.98-2.96)$ & .06 & $0.45(0.18-1.10)$ & .08 & $1.81(0.86-3.81)$ & .12 & $1.15(0.76-1.73)$ & .51 \\
\hline Depth $\geq 3.0 \mathrm{~mm}$ & $2.12(1.20-3.77)$ & .01 & $4.16(1.57-11.04)$ & .00 & $2.47(1.13-5.41)$ & .02 & $2.73(1.77-4.21)$ & .00 \\
\hline $\begin{array}{l}\text { Any mucosal } \\
\text { epithelial } \\
\text { involvement }\end{array}$ & $2.02(1.08-3.78)$ & .03 & $1.40(0.54-3.63)$ & .49 & $1.35(0.63-2.90)$ & .44 & $1.24(0.81-1.91)$ & .32 \\
\hline
\end{tabular}

Abbreviations: DM, distant metastasis; DSD, disease-specific death; HR, hazard ratio; LR, local recurrence; NM, nodal metastasis; OD, overall death.

Once independent prognostic factors were determined from multivariable modeling, different combinations of risk factors were evaluated in terms of distinctiveness, homogeneity, and monotonicity to determine the system with the best risk stratification (prognostic groupings without overlapping CIs). All risk factors were weighted evenly, because in this small initial study, HR CIs were not sufficiently narrow to take differences in hazards into account for different risk factors.

The alternative T-classification system, the BWVTC, was developed based on risk factors that were predictive of at least one end point of interest on multivariate analysis, namely lichen sclerosus, poor tumor differentiation, tumor diameter $\geq 2.0 \mathrm{~cm}$, tumor depth $\geq 3.0 \mathrm{~mm}$, and any mucosal epithelial involvement. In the BWVTC, class BWT1 tumors are those with none of the 5 risk factors, class BWT2 tumors have 1 high-risk factor, class BWT3 tumors have 2 high-risk factors, and class BWT4 tumors have $\geq 3$ high-risk factors.

Tables 3 and 5 summarize the distinctiveness, homogeneity, and monotonicity of the BWVTC versus the AJCC. A total of 20 tumors (9\%) were classified as BWT1, $50(22 \%)$ as BWT2, $65(29 \%)$ as BWT3, and $91(40 \%)$ as BWT4. Some overlap of CIs was observed for most BWVTC classes. However, most poor outcomes occurred in high BWVTC T classes (BWT3/BWT4). Specifically, $87 \%$ of LR, 92\% of NM, $91 \%$ of DM or DSD, and $78 \%$ of OD occurred in the BWT3/BWT4 group. Results of McNemar test confirmed superior homogeneity and monotonicity in the BWVTC classification system compared with the AJCC T-classification system for all poor outcomes of interest $(P<.001)$.

Kaplan-Meier curves and life tables for diseaserelated survival (LR, NM, DM, DSD) and overall survival for AJCC vulvar cancer and BWVTC T classes are shown in Figure 1. Pairwise comparison testing showed a significant difference between the lower classes of the AJCC system for disease-related survival (T1a vs T1b: $P=.01$; T1b vs T2: $P=.01$ ) and overall survival (T1b vs T2: $P=.01$ ). No difference was seen in the higher classes of the AJCC vulvar cancer Tclassification system for disease-related survival (T2 vs T3: $P=.50)$ and overall survival (T2 vs T3: $P=.17$ ),

\begin{tabular}{|c|c|c|c|c|c|c|c|c|c|}
\hline \multirow[b]{2}{*}{ Tumor Classification } & \multirow[b]{2}{*}{ N } & \multicolumn{2}{|c|}{ LR $(\mathrm{N}=65)$} & \multicolumn{2}{|c|}{$\mathrm{NM}(\mathrm{N}=24)$} & \multicolumn{2}{|c|}{ DM or DSD $(\mathrm{N}=35)$} & \multicolumn{2}{|c|}{$O D(N=99)$} \\
\hline & & $\mathrm{n}$ & $\%$ & $\mathrm{n}$ & $\%$ & $\mathrm{n}$ & $\%$ & $\mathrm{n}$ & $\%$ \\
\hline \multicolumn{10}{|l|}{ Homogeneity } \\
\hline AJCC T1a/T1b & 190 & 50 & 77 & 19 & 79 & 23 & 66 & 78 & 78 \\
\hline BWT1/BWT2 & 70 & 9 & 13 & 2 & 8 & 3 & 9 & 22 & 22 \\
\hline \multicolumn{10}{|l|}{ Monotonicity } \\
\hline AJCC T2/T3 & 36 & 15 & 23 & 5 & 21 & 12 & 34 & 22 & 22 \\
\hline BWT3/BWT4 & 156 & 56 & 87 & 22 & 92 & 32 & 91 & 77 & 78 \\
\hline
\end{tabular}

Abbreviations: BWT, Brigham and Women's tumor class; BWVTC, Brigham and Women's Vulvar Tumor Classification; DM, distant metastasis; DSD, disease-specific death; LR, local recurrence; NM, nodal metastasis; OD, overall death. 
Tumor Classification System for Vulvar SCC

whereas a significant difference was seen in the high BWVTC T classes (BWT3 vs BWT4: P<.001) for both survival curves.

\section{Discussion}

Our results indicate that AJCC 8th edition T-classification system for vulvar cancer suboptimally riskstratifies patients with vSCC, because most poor outcomes occur in the lower $\mathrm{T}$ classes. Risk factors independently associated with poor outcomes in this cohort are history of lichen sclerosus, tumor diameter $\geq 2.0 \mathrm{~cm}$, tumor depth $\geq 3.0 \mathrm{~mm}$, poorly differentiated histology, and mucosal epithelial involvement. The BWVTC, developed based on these prognostic factors, performs better than the AJCC T-classification system in terms of homogeneity and monotonicity. Most poor outcomes were shown to occur in the higher T classes (BWT3/BWT4), such as $92 \%$ of NM and $91 \%$ of DM/DSD. Conversely, in the AJCC T-classification system, most poor outcomes occur in the lower T classes, with only $21 \%$ of NM and $34 \%$ DM/DSD occurring in AJCC T2/T3 cases. Both the AJCC and BWVTC systems have some statistical overlap between $\mathrm{T}$ classes, indicating these classes may not be completely distinct. A larger study is likely needed to prove distinctiveness of T classes via Kaplan-Meier curves.

The prognostic factors identified in this study as independent predictors of poor outcomes are consistent with risk factors associated with recurrence and death in the published literature. Patients with untreated lichen sclerosus have a 10- to 100-fold increased risk of developing vSCC. ${ }^{17}$ Lichen sclerosus is a chronic inflammatory disease that can act as an initiator and promoter of carcinogenesis. ${ }^{18}$ The cutoff value of $2.0 \mathrm{~cm}$ for tumor diameter has been shown to be prognostically significant in multiple studies, including the present one. ${ }^{19,20}$ Shimm et a ${ }^{20}$ found that the $2.0-\mathrm{cm}$ cutoff had greater significance in survival than $3.0 \mathrm{~cm}$. In a Gynecologic Oncology Group study, groin NM occurred in $18.9 \%$ of tumors $<2.0 \mathrm{~cm}$ and $41.6 \%$ of tumors $>2.0 \mathrm{~cm} .{ }^{19}$ Deeper stromal invasion has been associated with NM, with the risk significantly increasing to $26.7 \%$ for tumors with stromal invasion $>3.0 \mathrm{~mm},{ }^{21}$ and this $3.0-\mathrm{mm}$ cutpoint proved to be the best prognostic cutpoint in the present study, as well. Histologic grade also has important prognostic implications, with 5-year survival rates for patients with well-differentiated, moderately differentiated, and poorly differentiated tumors reported to be $42 \%, 29 \%$, and $0 \%$, respectively. ${ }^{22}$ Anatomic differences between the cutaneous and mucosal surfaces of the vulva appear to impact prognosis in vulvar melanoma. ${ }^{23-25}$ Some studies, including the present one, have identified worse prognosis in vSCC when the mucosal site of the vulva is involved. ${ }^{6,8-10}$ This is thought to be due to the lack of a fat buffer layer beneath the epithelium, and subsequent rapid tumor access to larger lymphovascular channels in mucosal tissues, making metastasis more likely.

Heaps et $\mathrm{al}^{26}$ showed that no patients with a surgical margin clearance $\geq 8 \mathrm{~mm}$ developed LR compared with 21 cases of recurrence in those with a margin $<8 \mathrm{~mm}$. However, our study did not demonstrate an association between excisional margin or treatment modality and outcomes. Our data indicate that LAN was protective against NM and DM/DSD in patients with high T classes (BWT3/BWT4), with approximately 20\% declines in risk of NM and DM/DSD. Because tumor classification systems are based on tumor factors and not treatment factors, it was not appropriate to incorporate nodal evaluation into the T-classification system itself. However, the impact of LAN on outcomes of high-T-class vSCC merits further investigation.

This is the first study to evaluate the performance of the AJCC vulvar cancer T classification. Prior studies have assessed the performance of the International Federation of Gynecology and Obstetrics (FIGO) and the $\mathrm{N}$ classification systems for vulvar cancer. ${ }^{27,28}$ However, most vulvar cancers are localized at presentation, and therefore it is also important to evaluate the performance of T classification. ${ }^{3}$ The results from this study indicate that current AJCC T classification is not appropriately stratifying patients prognostically. Most poor outcomes occur in the low AJCC T1a and T1b classes (77\% of LR, $79 \%$ of NM, $66 \%$ of DM/DSD, and $78 \%$ of OD). The highest AJCC class (T3) poorly predicts poor outcomes, with only $2 \%$ of LR, $0 \%$ of NM, $6 \%$ of $\mathrm{DM} / \mathrm{DSD}$, and $3 \%$ of OD occurring in this class. The current AJCC T classification assigns these high-risk tumors (depth of stromal invasion $\geq 3.0 \mathrm{~mm}$ ) to class $\mathrm{T} 1 \mathrm{~b}$, likely accounting for the many poor outcomes in this group. Relatively few vSCC cases present initially with urethral, vaginal, or greater intrapelvic 
Le et al
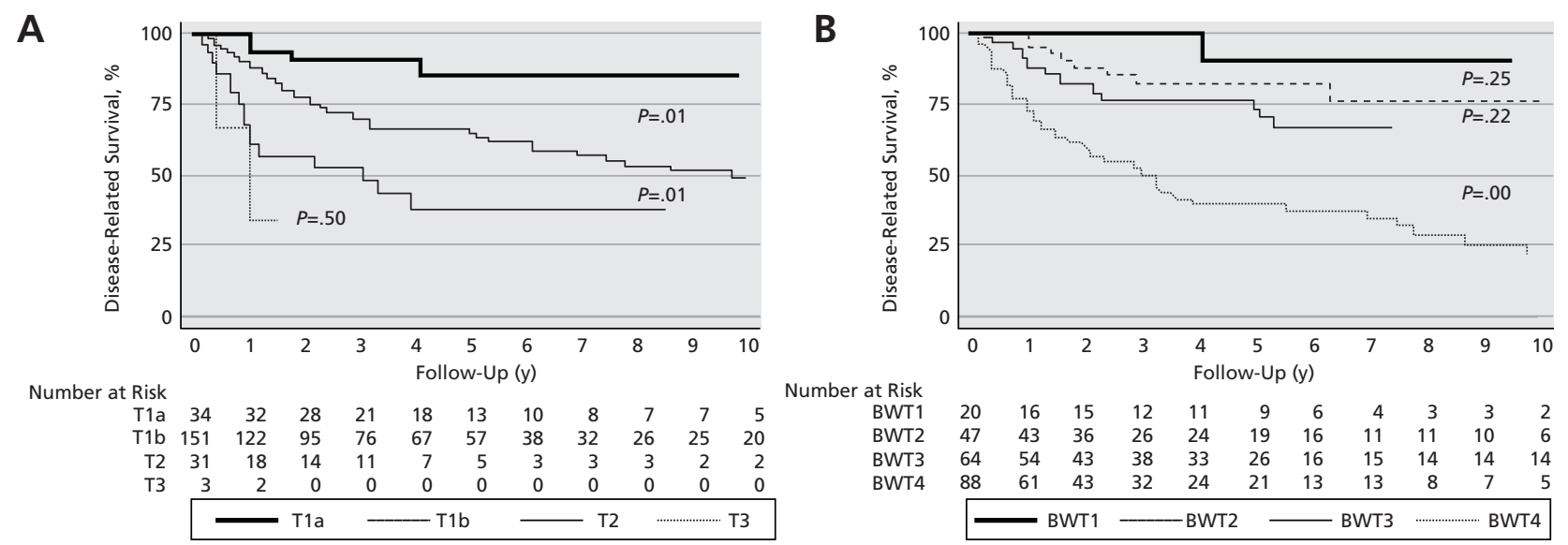

C


Figure 1. Kaplan-Meier survival curves for (A) disease-related survival by AJCC vulvar cancer tumor class (T), (B) disease-related survival by Brigham and Women's tumor class (BWT), (C) overall survival by AJCC vulvar cancer T class, and (D) overall survival by BWT class.

extension, and therefore few cases are in classes T2/ T3, driving poor outcomes into T1a/T1b. Only 36 of $226(16 \%)$ cases were in these upper classes in this tertiary center cohort.

The BWVTC T classification was developed with the goal of having a greater proportion of poor outcomes in the higher T classes, which would improve homogeneity and monotonicity with greater separation of high-risk and low-risk tumors. In contrast to AJCC T classification, only a small percentage of poor outcomes occurred in the BWT1 and BWT2 classes (13\% of LR, $8 \%$ of NM, $9 \%$ of DM/ DSD, and 22\% of OD). The lower BWVTC classes (T1/T2) had a $0 \%$ to $10 \%$ risk of NM, DM, or DSD compared with the higher incidences of these poor outcomes in BWT3 (17\%-19\%) and BWT4 $(32 \%-44 \%)$.
The focus of this study was on T classification, not $\mathrm{N}$ and $\mathrm{M}$ classification. Thus, the BWVTC system is not meant to replace TNM classification. However, it may be helpful to those designing future versions of AJCC T classifications and to tumor registries, such as the SEER database, which are designed to capture data on important prognostic factors. Meanwhile, because prognostic data are lacking for vSCC, the BWVTC may help clinicians estimate prognosis in patients with NOMO primary tumors and consider nodal staging and adjuvant treatment options, bearing in mind that the BWVTC was developed with data from a single center.

This study is subject to some limitations. The 2-center study design may have precluded full evaluation of some potentially important but relatively rare prognostic factors, such as immunosuppression, perineural invasion, and lymphovascular invasion. 
Tumor Classification System for Vulvar SCC

However, vSCC is itself a rare malignancy, and prospective studies with adequate enrollment would be difficult; only 226 patients met inclusion criteria at the 2 included sites over 15 years. Still, the data show that AJCC classification is not performing optimally. Multi-institutional retrospective studies will be needed to move vSCC classification forward and to validate both the AJCC and BWVTC T classifications. If such studies are performed and the gynecologic oncology community finds the BWVTC or a subsequent modification helpful, relevant risk factors could be routinely recorded in clinic notes. More precise documentation of risk factors will allow large registries, such as SEER, to collect data on these important prognostic factors, which would lead to further improvements in staging.

\section{Conclusions}

The goal of cancer classification is to separate patients into distinctive prognostic groups in order to aid clinicians in determining appropriate treatment regimens and to identify patients who may benefit from clinical trials. The proposed BWVTC classification offers increased homogeneity and monotonicity of $\mathrm{T}$ classification over the current AJCC vulvar cancer classification system. The BWVTC may allow clinicians to identify patients with high-risk vSCC who require more aggressive management, and identifies a low-risk group with a $\leq 10 \%$ risk of metastasis and death from disease. Further work is needed to optimize and validate vSCC classification. Meanwhile, the BWVTC appears to be an improvement over current AJCC vulvar cancer T classification.

\section{References}

1. Australian Institute of Health and Welfare. Gynaecological Cancers in Australia: An Overview. Available at: https://www.aihw.gov.au/reports/ cancer/gynaecological-cancers-in-australia-an-overview/contents/summary. Accessed December 1, 2017.

2. Ansink AC, Heintz AP. Epidemiology and etiology of squamous cell carcinoma of the vulva. Eur J Obstet Gynecol Reprod Biol 1993;48:111-115.

3. National Cancer Institute. Cancer Stat Facts: Vulvar Cancer. Available at: https://seer.cancer.gov/statfacts/html/vulva.html. Accessed December 1, 2017.

4. Hacker NF, Berek JS, Lagasse LD, et al. Management of regional lymph nodes and their prognostic influence in vulvar cancer. Obstet Gynecol 1983;61:408-412.

5. Homesley HD, Bundy BN, Sedlis A, et al. Assessment of current International Federation of Gynecology and Obstetrics staging of vulvar carcinoma relative to prognostic factors for survival (a Gynecologic Oncology Group study). Am J Obstet Gynecol 1991;164:997-1003; discussion 1003-1004.

6. Andreasson B, Nyboe J. Value of prognostic parameters in squamous cell carcinoma of the vulva. Gynecol Oncol 1985;22:341-351.

7. Aragona AM, Cuneo NA, Soderini AH, et al. An analysis of reported independent prognostic factors for survival in squamous cell carcinoma of the vulva: is tumor size significance being underrated? Gynecol Oncol 2014;132:643-648.

8. Boyce J, Fruchter RG, Kasambilides E, et al. Prognostic factors in carcinoma of the vulva. Gynecol Oncol 1985;20:364-377.

9. Hinten F, van den Einden LC, Cissen M, et al. Clitoral involvement of squamous cell carcinoma of the vulva: localization with the worst prognosis. Eur J Surg Oncol 2015;41:592-598.

10. Magrina JF, Gonzalez-Bosquet J, Weaver AL, et al. Primary squamous cell cancer of the vulva: radical versus modified radical vulvar surgery. Gynecol Oncol 1998; 71:116-121.

11. Raspagliesi F, Hanozet F, Ditto A, et al. Clinical and pathological prognostic factors in squamous cell carcinoma of the vulva. Gynecol Oncol 2006;102:333-337.

12. Sznurkowski JJ, Milczek T, Emerich J. Prognostic factors and a value of 2009 FIGO staging system in vulvar cancer. Arch Gynecol Obstet 2013;287:12111218.

13. Woolderink JM, de Bock GH, de Hullu JA, et al. Patterns and frequency of recurrences of squamous cell carcinoma of the vulva. Gynecol Oncol 2006;103:293-299.
14. $X u L Q$, Luo RZ, Sun $X M$, et al. Prognostic analysis of early-stage squamous cell carcinoma of the vulva. World J Surg Oncol 2013;11:20.

15. Amin MB, Edge S, Greene F, et al. AJCC Cancer Staging Manual, 8th ed. New York, NY: Springer; 2017.

16. Fine JP, Gray RJ. A proportional hazards model for the subdistribution of a competing risk. J Am Stat Assoc 1999;94:496-509.

17. Crum CP, McLachlin CM, Tate JE, et al. Pathobiology of vulvar squamous neoplasia. Curr Opin Obstet Gynecol 1997;9:63-69.

18. Carlson JA, Ambros R, Malfetano J, et al. Vulvar lichen sclerosus and squamous cell carcinoma: a cohort, case control, and investigational study with historical perspective; implications for chronic inflammation and sclerosis in the development of neoplasia. Hum Pathol 1998;29:932-948.

19. Homesley HD, Bundy BN, Sedlis A, et al. Prognostic factors for groin node metastasis in squamous cell carcinoma of the vulva (a Gynecologic Oncology Group study). Gynecol Oncol 1993;49:279-283.

20. Shimm DS, Fuller AF, Orlow EL, et al. Prognostic variables in the treatment of squamous cell carcinoma of the vulva. Gynecol Oncol 1986;24:343-358.

21. Hacker NF, Van der Velden J. Conservative management of early vulvar cancer. Cancer 1993;71(Suppl 4):1673-1677.

22. Nicoletto MO, Parenti A, Del Bianco P, et al. Vulvar cancer: prognostic factors. Anticancer Res 2010;30:2311-2317.

23. Chang AE, Karnell LH, Menck HR. The National Cancer Data Base report on cutaneous and noncutaneous melanoma: a summary of 84,836 cases from the past decade. The American College of Surgeons Commission on Cancer and the American Cancer Society. Cancer 1998;83:1664-1678.

24. Lotem M, Anteby S, Peretz T, et al. Mucosal melanoma of the female genital tract is a multifocal disorder. Gynecol Oncol 2003;88:45-50.

25. Mihajlovic M, Vlajkovic S, Jovanovic P, et al. Primary mucosal melanomas: a comprehensive review. Int J Clin Exp Pathol 2012;5:739-753.

26. Heaps JM, Fu YS, Montz FJ, et al. Surgical-pathologic variables predictive of local recurrence in squamous cell carcinoma of the vulva. Gynecol Oncol 1990;38:309-314.

27. Baiocchi G, Silva Cestari FM, Rocha RM, et al. Prognostic value of the number and laterality of metastatic inguinal lymph nodes in vulvar cancer: revisiting the FIGO staging system. Eur J Surg Oncol 2013;39:780-785.

28. Li J, Cai Y, Ke G, et al. Validation of the new FIGO staging system (2009) for vulvar cancer in the Chinese population. Gynecol Oncol 2015;137:274-279.

See JNCCN.org for supplemental online content. 